\title{
Factores sectoriales y regionales que determinan la dinámica empresarial en los países en desarrollo: evidencia sobre las actividades manufactureras de tecnología baja, media y alta en la Argentina
}

\author{
Carla Daniela Calá ${ }^{1}$
}

\section{Resumen}

\begin{abstract}
En este artículo se analizan los determinantes de la dinámica empresarial en los países en desarrollo, tomándose como ejemplo la Argentina. Se explica la entrada y salida de las empresas a nivel regional y distinguen actividades manufactureras de tecnología baja, media y alta. Se concluye que hay factores regionales y sectoriales que explican esa dinámica, aunque el efecto no es homogéneo en todos los sectores. En los sectores de tecnología baja son particularmente relevantes las variables explicativas características de las economías en desarrollo (pobreza, economía informal y capacidad ociosa). Existe un patrón centro-periferia por el que las economías de aglomeración y las entradas y salidas anteriores tienen efectos diferentes en la región central o periférica. Los encargados de formular políticas en los países en desarrollo deberían tener en cuenta no solo las particularidades de esas economías, sino también la heterogeneidad regional en materia de desarrollo y composición industrial del país.
\end{abstract}

\section{Palabras clave}

Empresas industriales, empresas manufactureras, países en desarrollo, estudios de casos, desarrollo de empresas, datos estadísticos, modelos econométricos, Argentina Clasificación JEL

R12, R30, C33

\section{Autora}

Carla Daniela Calá es docente e investigadora en la Facultad de Ciencias Económicas y Sociales de la Universidad Nacional de Mar del Plata, Argentina. Correo electrónico: dacala@mdp.edu.ar. 


\section{Introducción}

Hay numerosas publicaciones sobre los factores que determinan la entrada y la salida de las empresas manufactureras. Este interés se explica no solo por las consecuencias directas que las empresas nuevas tienen en el empleo y la producción, sino también por los efectos indirectos que ejercen en la eficiencia del mercado, la productividad empresarial, la innovación y, en última instancia, el crecimiento económico (Audretsch y Keilbach, 2005). Sin embargo, esta literatura no se ha complementado lo suficiente con estudios sobre los factores determinantes sectoriales que permitan explicar el hecho de que los factores regionales que determinan la creación de empresas nuevas varían entre los sectores manufactureros. Así pues, tras la labor fundamental de Audretsch y Fritsch (1999), en varios estudios se han tenido en cuenta las dimensiones regionales y sectoriales a fin de evaluar debidamente el efecto de las características regionales sobre la dinámica empresarial y considerar la heterogeneidad en distintos niveles ${ }^{2}$. Esos estudios confirman que los factores regionales que determinan la creación de empresas nuevas difieren entre los sectores manufactureros (Carree, Verheul y Santarelli, 2011) y que ciertas condiciones regionales pueden estimular dicha creación en algunos sectores, pero desalentarla en otros. Como resultado de ello, algunos instrumentos de política pueden alentar la creación de empresas, pero no necesariamente en los tipos de sectores que los encargados de formular las políticas pretenden (Audretsch y Fritsch, 1999).

Cabe señalar que los estudios sobre los factores regionales y sectoriales que determinan la entrada y la salida de las empresas se circunscriben a los países desarrollados. La investigación que se centra en los países en desarrollo es mucho menor, a pesar de que se prevé que esos países se conviertan en actores fundamentales de la economía mundial, pues la opinión generalizada es que su participación en la producción aumentará de forma considerable en los próximos decenios (Wilson y Purushothaman, 2006). Más concretamente, los estudios sobre los países en desarrollo se centran, o bien en los factores determinantes sectoriales (Lay, 2003; Wang, 2006; Günalp y Cilasun, 2006; Ozturk y Kilic, 2012), o bien en los regionales (Naudé y otros, 2008; Santarelli y Tran, 2012; Calá, Arauzo-Carod y Manjón-Antolín, 2015; Calá, Manjón-Antolín y Arauzo-Carod, 2016). Por lo tanto, el presente parecería ser el primer intento de cuantificar los factores regionales que determinan la entrada y la salida de las empresas en distintos sectores manufactureros de un país en desarrollo.

El objetivo de este estudio es proporcionar información útil dirigida a los encargados de formular políticas en los países en desarrollo que estén interesados en diseñar políticas públicas que promuevan la creación (y la supervivencia) de empresas nuevas en todo el país. Con ese fin, se utiliza la Argentina como caso ilustrativo para explicar la entrada y la salida de las empresas a nivel regional, y se hace una distinción entre tres grupos de actividades manufactureras: las de tecnología baja, media y alta. En primer lugar, se evalúa si los factores sectoriales y regionales que determinan la entrada y la salida difieren entre los sectores. En segundo lugar, se tienen en cuenta las particularidades de los países en desarrollo mediante la incorporación de factores propios de estos, como los niveles de pobreza, el tamaño del sector informal, la capacidad ociosa o la heterogeneidad estructural de las regiones.

En el estudio se llega a la conclusión de que hay factores regionales y sectoriales que explican la dinámica empresarial, pero que su efecto no es homogéneo en todos los grupos de sectores. En particular, las variables que constituyen un indicador de las características propias de la Argentina como país en desarrollo (el nivel de pobreza, el tamaño del sector informal y la capacidad ociosa) afectan sobre todo la entrada y la salida de las empresas de baja tecnología. También hay pruebas de que existe un patrón de centro-periferia que se observa en todos los grupos de sectores. Estos resultados sugieren que, en las políticas de creación de empresas de los países en desarrollo, se deberían tener

\footnotetext{
2 Estos estudios son: Arauzo-Carod y otros (2007), que se centra en España; Carree, Verheul y Santarelli (2011), sobre Italia; Fotopoulos y Spence (1998), en relación con Grecia; Fritsch y Falck (2007), sobre Alemania; Nurmi (2006), acerca de Finlandia, y Nyström (2007), sobre Suecia.
} 
en cuenta no solo las particularidades de esas economías, sino también la heterogeneidad regional, tanto en lo que respecta al nivel de desarrollo como a la composición industrial en el país.

El presente artículo se divide en cinco secciones. En la sección que figura a continuación se examina brevemente la literatura empírica sobre los factores regionales que determinan la entrada y la salida de las empresas en las economías desarrolladas y en desarrollo. También se examinan los motivos por los que algunos factores regionales pueden tener un efecto distinto en los países desarrollados y en los países en desarrollo. En la sección III se describe la dinámica empresarial de la Argentina durante el período de interés, así como el conjunto de datos, la estrategia empírica y el modelo econométrico. En la sección IV se presentan los principales resultados y, en la sección V, la conclusión.

\section{Factores que determinan la entrada y la salida de las empresas: teoría y datos}

\section{Países desarrollados}

En la literatura se considera que las diferencias entre algunas características de las regiones explican las variaciones considerables entre las pautas de entrada y salida que se observan en ellas. Dichas características son los mercados de trabajo, la estructura industrial y la concentración espacial de las actividades económicas y las personas ${ }^{3}$.

En cuanto a las diferencias en el mercado de trabajo, la literatura se ha centrado en los efectos del desempleo, los salarios y el nivel educativo de la fuerza de trabajo. En primer lugar, se observa que no hay consenso sobre la forma en que el desempleo afecta la dinámica empresarial. Según la hipótesis de la incitación (push hypothesis), el desempleo tiene un efecto positivo en la entrada de empresas en la medida en que las personas desempleadas pueden crear empresas nuevas. Asimismo, cuando el desempleo aumenta, los trabajadores autónomos tienen menos oportunidades de empleo, por lo que sus empresas son menos proclives a la salida (Carree y Thurik, 1996; Nyström, 2007; Carree, Santarelli y Verheul, 2008; Santarelli, Carree y Verheul, 2009). Por el contrario, según la hipótesis de la disuasión (pull hypothesis), un aumento del desempleo puede restringir la entrada, ya que los desocupados carecen de competencias empresariales y de capital. Además, dado que la desocupación es un indicador del nivel de actividad económica, una mayor tasa de desempleo puede dar lugar a un aumento del número de salidas (Brixy y Grotz, 2007). En segundo lugar, en lo que respecta a los salarios, el aumento del costo de la mano de obra desalienta la entrada de nuevas empresas y favorece la salida (Santarelli, Carree y Verheul, 2009). En tercer lugar, la disponibilidad de mano de obra calificada puede fomentar la entrada de nuevas empresas en los sectores que requieren competencias específicas (Spilling, 1996) ${ }^{4}$.

En cuanto a la estructura industrial, los estudios anteriores se han centrado en el nivel de diversificación de la industria, la tradición industrial, la proporción de pequeñas y medianas empresas (pymes), y la relación entre las entradas y las salidas. Los entornos más diversificados promueven la entrada de nuevas empresas y su supervivencia, ya que aumentan las posibilidades de que los

\footnotetext{
3 También hay una serie de factores que, si bien son importantes para explicar la dinámica empresarial, no se pueden incluir con facilidad en los análisis empíricos. Este es el caso de las actitudes culturales respecto de la iniciativa empresarial (Shapero, 1983), así como el papel que desempeña el gobierno mediante el gasto público en infraestructura o las políticas públicas (Reynolds, Storey y Westhead, 1994).

4 Debe tenerse en cuenta que las personas que poseen un gran capital humano son mejores para descubrir y aprovechar las oportunidades comerciales, pero al mismo tiempo es más probable que tengan empleos bien remunerados y no son necesariamente más proclives a crear nuevas empresas (Nyström, 2007).
} 
recursos se reasignen a nuevas actividades cuando ocurre una perturbación negativa (Kosacoff y Ramos, 1999). Además, la tradición industrial puede impulsar las actividades empresariales en curso (Rocha y Sternberg, 2005) y desalentar el cierre de las empresas, dado que es probable que las empresas continuadoras anteriores hayan creado un entorno comercial favorable e instituciones de apoyo. Cabe esperar que la proporción de pymes aumente las turbulencias regionales, ya que promueve tanto la entrada como la salida. Por una parte, los costos asociados con la entrada son más bajos en las zonas donde hay una densa red de pymes, que pagan salarios inferiores (con lo que se reduce el costo de oportunidad del empleo por cuenta propia) y sirven de ejemplo para los nuevos empresarios (Audretsch, 1995b). Por otra parte, dado que las pequeñas empresas tienen más probabilidades de salir debido a las desventajas que enfrentan en materia de costos, la cantidad de salidas debería ser mayor en las regiones donde hay una gran proporción de empresas pequeñas (Fotopoulos y Spence, 1998). Lo anterior está estrechamente vinculado con la relación entre las entradas y las salidas. Las entradas pueden incidir en las salidas porque aumentan la presión de la competencia en el mercado (lo que se conoce como efecto de desplazamiento) y, al mismo tiempo, las empresas que abandonan el mercado dejan nichos de consumidores insatisfechos, lo que alienta a nuevas empresas a ingresar (efecto de sustitución). En particular, según el fenómeno de la puerta giratoria, muchas empresas (pequeñas) salen solo algunos años después de su creación (Audretsch, 1995a).

Además, en las zonas de mayor concentración suele haber más entradas y menos salidas, porque las empresas se benefician de las economías externas locales, como la especialización de los proveedores, la elevada densidad del mercado de trabajo y los beneficios tecnológicos indirectos, así como de la proximidad física de los consumidores (Armington y Acs, 2002; Keeble y Walker, 1994; Littunen, Storhammar y Nenonen, 1998; Reynolds, Storey y Westhead, 1994). No obstante, pueden presentarse economías de desaglomeración que obstaculicen la entrada y den lugar a un aumento de la salida. Ello se debe a que la mayor densidad eleva el precio de los insumos al aumentar la competencia por recursos escasos ${ }^{5}$.

Sin embargo, es probable que el efecto de estas características regionales difiera entre los sectores. Por ejemplo, según la teoría del ciclo de vida de los productos (Vernon, 1966), las nuevas empresas innovadoras obtienen más ventajas de las economías de aglomeración en las primeras etapas de su existencia, dado que en las zonas urbanas densas se accede mejor al capital, la mano de obra calificada, la infraestructura, la información y las oportunidades de interactuar con otras empresas. A medida que los productos de las nuevas empresas maduran, la competencia entre ellas se basa en el ofrecimiento de precios más bajos, lo que les exige reducir el costo de sus insumos. Además, el efecto de los factores regionales, como el nivel de ingresos o el desempleo, puede depender de la elasticidad de la demanda o del grado de intensidad del capital, respectivamente (Audrestch y Fritsch, 1999). El hecho de que en la literatura pertinente se presenten resultados desiguales y parcialmente contradictorios tal vez se deba a que se han ignorado esas diferencias entre los sectores (Audrestch y Fritsch, 1999; Fritsch y Falck, 2007).

Esta limitación solo se ha abordado en unos pocos estudios ${ }^{2}$, en los que se han tenido en cuenta, además de las variables regionales, algunos factores propios de cada sector. En particular, en estos estudios se incluyen las barreras a la entrada y la salida, y se llega a la conclusión de que la importancia relativa de los factores propios de cada ubicación es mayor en los sectores donde las barreras son menores (Arauzo-Carod y otros, 2007; Fotopoulos y Spence, 1998; Fritsch y Falck, 2007; Nurmi, 2006). En dichos estudios también se muestra que, si bien la demanda de productos propios

\footnotetext{
5 También puede haber una mayor cantidad de salidas en las zonas que tienen una gran densidad de población (véanse algunos datos empíricos en, por ejemplo, Buss y Lin, 1990, Forsyth, 2005 y Huiban, 2011). Existen varios motivos que explican esto, entre ellos: la mayor competencia en el mercado de bienes y el de factores (Agarwal y Gort, 1996; Bresnahan y Reiss, 1991); las mayores posibilidades de encontrar empleo, hallar otra oportunidad empresarial o vender los activos de las empresas a otros emprendimientos (Huiban, 2011), y, como se examina más adelante, el hecho de que las zonas urbanas atraigan más entradas, lo que conduce a una mayor proporción de empresas jóvenes que puede conllevar una mayor cantidad de salidas.
} 
de cada sector es un factor clave en algunos de ellos, hay otras actividades que dependen más de la evolución de la demanda total (regional y nacional). Además, no está claro de qué modo el número de empresas continuadoras en el mismo sector afecta la dinámica empresarial. Por una parte, dicho número puede atraer a empresas similares que se benefician de las externalidades positivas (conocidas como economías de localización); por otra parte, puede ejercer un efecto de competencia que impida la entrada y aumente la salida (Carree, Verheul y Santarelli, 2011).

\section{Países en desarrollo}

Aunque hay muy pocos datos empíricos sobre los factores que determinan la entrada y la salida de las empresas en los países en desarrollo, la cantidad de información está aumentando con rapidez. En particular, Lay (2003) y Wang (2006) analizan la entrada de nuevas empresas utilizando datos de la Provincia China de Taiwán a nivel sectorial, y lo mismo se hizo en Turquía (Günalp y Cilasun, 2006; Ozturk y Kilic, 2012). Calá, Manjón-Antolín y Arauzo-Carod (2016), Naudé y otros (2008), y Santarelli y Tran (2012) emplean datos a escala regional en relación con la Argentina, Sudáfrica y Viet Nam, respectivamente. También se han estudiado los factores que determinan la salida en algunos países: Ozturk y Kilic (2012) y Lay (2003) utilizan datos de Turquía y la Provincia China de Taiwán a nivel sectorial; Calá, Arauzo-Carod y Manjón-Antolín (2015) emplean datos regionales de la Argentina, y Frazer (2005) y Eslava y otros (2006) usan datos a nivel de las empresas para estudiar los casos de Ghana y Colombia, respectivamente, mientras que Álvarez y Vergara (2010 y 2013) y López (2006) hacen lo mismo en relación con Chile.

Es interesante señalar que hay varias características de las economías en desarrollo que pueden afectar la dinámica empresarial y los factores que la determinan, lo que pone de relieve la necesidad de hacer investigaciones empíricas concretas (Bruton, Ahlstrom y Obloj, 2008). En primer lugar, los países en desarrollo suelen caracterizarse por la inestabilidad macroeconómica y las variaciones cíclicas intensas (Stiglitz, 1998; Ocampo, Rada y Taylor, 2009). Por lo tanto, cabe esperar que sean más vulnerables a las perturbaciones macroeconómicas. Esto significa que, tras cada crisis, un gran número de empresas ingresan en los mercados en crecimiento, pero muchas de ellas salen en los años subsiguientes. Por lo tanto, el fenómeno de la puerta giratoria puede ocurrir con mayor intensidad de la que suele observarse en los países desarrollados ${ }^{6}$. Además, después de una crisis económica, las empresas establecidas que explotan su capacidad ociosa pueden tener un mayor efecto global que las nuevas empresas sobre la satisfacción de la demanda de nuevos productos (Calá, Manjón-Antolín y Arauzo-Carod, 2016).

Además, la volatilidad macroeconómica puede mitigar el efecto de variables como la tasa de desempleo o la tradición industrial. Las personas que han estado cesantes durante períodos prolongados pueden no tener la capacidad, los recursos financieros ni el capital social necesario para crear una nueva empresa (Fritsch y Falck, 2007). Por otra parte, los cambios de las condiciones que determinan la rentabilidad (el tipo de cambio, los aranceles aduaneros, el acceso al crédito o la política tributaria) y la falta de continuidad de las políticas industriales pueden reducir el efecto que las economías de localización (dinámicas) anteriores tienen sobre la entrada y la salida en la actualidad (Calá, Manjón-Antolín y Arauzo-Carod, 2016).

El hecho de que la estructura industrial esté menos desarrollada y los mercados menos saturados puede afectar la relación entre las entradas y las salidas. Por ejemplo, se han hallado indicios que permiten rechazar el efecto de sustitución en los casos de Turquía y la Provincia China de Taiwán (Günalp y Cilasun, 2006; Lay, 2003). Además, las salidas pueden reflejar en realidad las

\footnotetext{
6 Sin embargo, esto puede no ocurrir si la menor competencia y el mayor poder monopolístico debilitaran la tasa de rotación de empresas y desaceleraran el proceso de destrucción creativa.
} 
expectativas negativas respecto de la evolución de la actividad económica, que disuaden la entrada (Calá, Manjón-Antolín y Arauzo-Carod, 2016). Del mismo modo, la aglomeración no siempre se asocia de forma positiva con el ritmo de creación de nuevas empresas, ya que la mayor competencia y el aumento de las barreras que obstaculizan la entrada pueden actuar como factores que disuaden la actividad empresarial en las regiones centrales (Naudé y otros, 2008).

Además, en los países en desarrollo el sector informal suele ser de gran tamaño (Schneider, 2005). En el plano regional, la relación entre el tamaño de la economía sumergida y la tasa de entrada puede ser positiva o negativa. Será positiva si las actividades de subcontratación crean complementariedades o si el sector informal fomenta la entrada al actuar como trampolín (Bennett, 2010) para que los empresarios puedan "tantear el terreno" antes de decidir si desean ingresar al sector formal. Además, la informalidad puede alentar la creación de empresas porque la inestabilidad y la inseguridad propias de los empleos informales hacen que las personas tengan más iniciativa empresarial. Sin embargo, es probable que la relación sea negativa si las empresas informales compiten con las formales sobre la base de precios inferiores o productos no diferenciados. En lo que respecta a las salidas, puede surgir una relación positiva si las empresas formales compiten con las empresas informales por los mismos recursos o se vuelven informales cuando se encuentran en situaciones difíciles. Por el contrario, cabe esperar una relación negativa si las empresas formales compran insumos al sector informal a fin de reducir los costos o aumentar la flexibilidad.

Otra característica distintiva de las economías en desarrollo es el elevado nivel de pobreza y la disparidad de los ingresos, tanto entre las personas como entre las regiones. Ello puede obstaculizar la aparición de nuevas empresas (formales), ya que la demanda de productos y servicios es inferior, menos estable y menos diversa. La pobreza afecta también la dotación de empresarios, ya que es limitada la proporción de personas que tienen acceso a información, redes empresariales y recursos financieros (Kantis, Angelelli y Moori Koening, 2005).

Por último, en los países en desarrollo se observan marcadas diferencias regionales en algunos indicadores económicos fundamentales, y hay algunas zonas donde el nivel de capitalización, tecnología, productividad y requisitos de capital humano se asemeja al de las regiones de los países avanzados (Sunkel, 1978). Una consecuencia directa de esta "heterogeneidad estructural" (Cassiolato, Pagola y Lastres, 2009) es que los factores que determinan la entrada y la salida de las empresas pueden diferir entre las regiones de un país. Por ejemplo, en las zonas periféricas por lo general no existe la masa crítica de empresas conexas que permitiría crear las condiciones para que hubiera economías externas en algunos sectores. Como resultado de ello, es de esperar que los efectos positivos de la aglomeración solo surjan en las zonas centrales. En estudios anteriores sobre las entradas y las salidas totales en la Argentina se ha concluido que la distribución espacial de dichas entradas y salidas muestra un patrón de centro-periferia (Calá, Arauzo-Carod y Manjón-Antolín, 2015 y 2016).

\section{La dinámica empresarial en la Argentina}

\section{Estrategia y datos empíricos}

Con el fin de determinar qué características regionales afectan la entrada y la salida de las empresas, en el estudio se desarrollan diferentes ecuaciones relativas al número de entradas (y salidas) en tres grupos de sectores manufactureros: i) de baja tecnología; ii) de tecnología media, y iii) de alta tecnología. A continuación se proporciona la fórmula general de estas ecuaciones:

$$
\operatorname{ENTRADA}_{i j t}=f\left(R E G I O N_{i t} ; S E C T O R_{i j t} ; M A C R O_{t}\right)
$$




$$
S A L I D A_{i j t}=f\left(R E G I O N_{i t} ; S E C T O R_{i j t} ; M A C R O_{t}\right)
$$

donde $R E G I O N_{i t}$ denota un grupo de factores propios de cada región que varían por año y por provincia; $S E C T O R_{i j t}$ se refiere a factores propios de cada sector que varían por provincia, año y grupo de sectores, y $M A C R O_{t}$ se refiere a factores a escala nacional que solo varían por año.

A fin de comprobar si los factores que determinan la dinámica empresarial en los países desarrollados tienen la misma importancia en la Argentina, se toma como punto de partida un conjunto de factores que se suelen hallar como explicativos de las entradas y las salidas regionales en esas economías, tanto en el plano sectorial como en el regional. Esto permite obtener un primer análisis de las diferencias entre los países desarrollados y los países en desarrollo (véanse algunas estrategias similares en Fritsch, Brixy y Falck, 2006, y Ghani, Kerr y O'Connell, 2014). Sobre la base de la información que figura en la sección II.2, cabe esperar que algunas variables que explican la entrada y la salida de las empresas en los países desarrollados tengan poca significación estadística o sean del signo contrario.

A continuación, en el estudio se examinan factores como el tamaño de la economía informal o el nivel de pobreza, que posiblemente sean importantes en los países en desarrollo, pero que nunca se toman en cuenta en los estudios sobre los países desarrollados. Por último, se procura determinar si existe una estructura de centro-periferia, para lo cual se incluye el resultado de multiplicar una variable ficticia que identifica las provincias más ricas, con variables que se espera que tengan efectos diferentes en las regiones centrales y no centrales (efectos de aglomeración y efectos de sustitución o desplazamiento). Esta es la segunda evaluación de las diferencias entre los países desarrollados y los países en desarrollo. Uno de los resultados previstos es que las variables en las que se incorporan algunas de las particularidades de los países en desarrollo tengan una capacidad explicativa sustancial. Otro es que los productos cruzados tengan efectos distintos en las regiones centrales y en las rezagadas.

\section{Entrada y salida}

Los datos relativos a las entradas y las salidas se tomaron del Observatorio de Empleo y Dinámica Empresarial (OEDE) del Ministerio de Trabajo y Seguridad Social de la Argentina. La base de datos contiene el número de entradas, salidas y empresas continuadoras sobre la base de todas las empresas manufactureras (formales y privadas) que tienen al menos un empleado inscrito en la seguridad social. Por consiguiente, el conjunto de datos no contiene información sobre el empleo público ni el informal. Además, el OEDE considera modificaciones del código de las empresas que no reflejan verdaderas entradas y salidas del mercado, porque una empresa se considera cerrada si no ha declarado empleados durante 12 meses consecutivos. No obstante, las salidas espurias causadas por el desplazamiento de toda la fuerza de trabajo de las empresas que "salen" para convertirse en "nuevas" empresas se identifican y se excluyen de la base de datos. Por último, el análisis se limita a las empresas manufactureras que declaran que la mayor parte de su fuerza de trabajo se encuentra en las regiones examinadas (aproximadamente el 90\% de todas las empresas en 2008). Por consiguiente, se excluyen las sucursales o las filiales ubicadas en otras regiones. En términos generales, de la información disponible en la actualidad para llevar a cabo estudios sobre la demografía empresarial en la Argentina, los datos que proporciona el OEDE son los que están más actualizados, son más integrales, se encuentran más desglosados en el plano espacial y abarcan un plazo razonablemente prolongado.

Se dispone de datos sobre las 23 provincias argentinas y la capital federal, Buenos Aires; la provincia que lleva este último nombre se divide en el Gran Buenos Aires y el resto de la provincia. La provincia de Río Negro se excluyó del estudio debido a la falta de datos sobre la mayoría de las 
variables explicativas. Por consiguiente, si bien en la base de datos hay 25 regiones, solo se obtienen resultados en relación con 24. Las actividades manufactureras se dividen en 23 sectores de dos dígitos, sobre la base de la taxonomía que sugirieron Katz y Stumpo (2001) y que posteriormente Katz y Bernat (2011) adaptaron para obtener una desagregación a dos dígitos (2011) ${ }^{7}$. Estos sectores se agrupan en tres categorías - de tecnología alta media y baja- de acuerdo con su grado de intensidad tecnológica (véase el anexo A1).

La variable dependiente es el número de entradas y salidas anuales en cada región y grupo de sectores durante el período que va de 2003 a 2008. Gracias a que el análisis se lleva a cabo a partir de 2003, en el estudio se excluye la ruptura estructural causada por la crisis económica y política de finales de 2001, que dio lugar a la devaluación del peso argentino en enero de 2002. De este modo se evita distorsionar los resultados por completo. El período de análisis termina en 2008, que era el último año disponible en la base de datos del OEDE en aquel momento. En el cuadro 1 se muestra la evolución de las entradas, las salidas y las empresas continuadoras en el período de análisis.

\section{Cuadro 1}

Argentina: entradas, salidas y empresas continuadoras, 2003-2008

(En número de empresas y en porcentajes)

\begin{tabular}{lccccc}
\hline Año & Entradas & Salidas & Continuadoras & Tasa de entrada $^{\text {a }}$ & Tasa de salida $^{\text {b }}$ \\
\hline 2003 & 4986 & 2330 & 42754 & 11,7 & 5,4 \\
\hline 2004 & 5994 & 2326 & 45234 & 13,3 & 5,1 \\
\hline 2005 & 5486 & 2929 & 48317 & 11,4 & 6,1 \\
\hline 2006 & 6264 & 3623 & 49987 & 12,5 & 7,2 \\
\hline 2007 & 5886 & 4358 & 51796 & 11,4 & 8,4 \\
\hline
\end{tabular}

Fuente: Elaboración propia, sobre la base de datos del Observatorio de Empleo y Dinámica Empresarial.

a La tasa de entrada se calcula como el número de entradas sobre el número de empresas continuadoras.

b La tasa de salida se calcula como el número de salidas sobre el número de empresas continuadoras.

El número elevado de entradas en el período que va de 2003 a 2005 está estrechamente relacionado con la recuperación de la economía argentina después de la crisis de 2001 y 2002. En el cuadro 1 se muestra que la elevada tasa de entrada que se observa desde 2003 (alrededor del $12 \%$ ) disminuye solo al final del período, pero sigue siendo alta (alrededor del 10\%). En cuanto a las salidas, sin embargo, después de los dos primeros años de estabilidad (2003 y 2004), se observó la tendencia opuesta, con una tasa de variación anual media del 17\%. Según el Ministerio de Trabajo, Empleo y Seguridad Social (2007), esto se debió en gran medida a la aparición de nuevas empresas en los años inmediatamente posteriores a la crisis (tanto a proyectos que se habían aplazado como a emprendimientos totalmente nuevos alentados por las mejores condiciones macroeconómicas). Además, la desaceleración de la entrada neta en 2008 se explica por la crisis financiera internacional, la apreciación gradual del tipo de cambio real y las tensiones internas (Katz y Bernat, 2011).

En el cuadro 2 se muestra que la distribución espacial de las empresas continuadoras, las entradas y las salidas no es homogénea, ya que la mayoría se concentran en las cinco regiones más ricas (la capital federal, el Gran Buenos Aires, el resto de la provincia de Buenos Aires, Santa Fe y Córdoba). En particular, alrededor del $80 \%$ de los trabajadores, las empresas continuadoras, las nuevas empresas y las empresas salientes se concentran en esas regiones, que abarcan aproximadamente el 22\% de la superficie del país. La distribución espacial desigual de la actividad económica es bastante característica de las economías en desarrollo (Scott y Storper, 2007).

\footnotetext{
7 Esta clasificación está basada en el recurso que se utiliza con más intensidad para producir los bienes: los recursos naturales, la mano de obra o la ingeniería. Ha sido adoptada por la CEPAL y es muy utilizada en los estudios sobre América Latina (CEPAL, 2007), pero difiere ligeramente de la que define la Organización de Cooperación y Desarrollo Económicos (OCDE).
} 


\section{Cuadro 2}

Argentina: empresas continuadoras, entradas y salidas por grupo de sectores manufactureros en la región central y la periférica, 2003-2008

(En número de empresas y en porcentajes)

\begin{tabular}{|c|c|c|c|c|}
\hline \multicolumn{5}{|c|}{ A. Promedio de empresas continuadoras por grupo de sectores en la región central y la periférica } \\
\hline & \multicolumn{2}{|c|}{ Número de empresas } & \multicolumn{2}{|c|}{ Porcentaje } \\
\hline & Periferia & Centro & Periferia & Centro \\
\hline Baja tecnología & 6534 & 22102 & 74,8 & 56,0 \\
\hline Tecnología media & 1420 & 9849 & 16,3 & 24,9 \\
\hline Alta tecnología & 776 & 7548 & 8,9 & 19,1 \\
\hline Total & 8730 & 39500 & 100,0 & 100,0 \\
\hline \multicolumn{5}{|c|}{ B. Entrada de empresas por grupo de sectores en la región central y la periférica } \\
\hline & \multicolumn{2}{|c|}{ Número de empresas } & \multicolumn{2}{|c|}{ Porcentaje } \\
\hline & Periferia & Centro & Periferia & Centro \\
\hline Baja tecnología & 5071 & 16805 & 76,4 & 62,2 \\
\hline Tecnología media & 1113 & 6107 & 16,8 & 22,6 \\
\hline Alta tecnología & 454 & 4098 & 6,8 & 15,2 \\
\hline Total & 6638 & 27010 & 100,0 & 100,0 \\
\hline \multicolumn{5}{|c|}{ C. Salida de empresas por grupo de sectores en la región central y la periférica } \\
\hline & \multicolumn{2}{|c|}{ Número de empresas } & \multicolumn{2}{|c|}{ Porcentaje } \\
\hline & Periferia & Centro & Periferia & Centro \\
\hline Baja tecnología & 3088 & 10754 & 78,3 & 65,1 \\
\hline Tecnología media & 576 & 3421 & 14,6 & 20,7 \\
\hline Alta tecnología & 279 & 2336 & 7,1 & 14,1 \\
\hline Total & 3943 & 16511 & 100,0 & 100,0 \\
\hline
\end{tabular}

Fuente: Elaboración propia, sobre la base de datos del Observatorio de Empleo y Dinámica Empresarial.

Nota: Las cifras son datos poblacionales.

Además, la composición de las empresas continuadoras, las entradas y las salidas también difiere. En las provincias centrales, la importancia relativa de los sectores de tecnología media y alta es mayor que en las regiones periféricas (véase el cuadro 2). Esto guarda relación con las ventajas que las provincias centrales ofrecen a estos tipos de actividades. En 2003, estas cinco regiones representaban en conjunto el $75 \%$ del gasto en ciencia y tecnología, el $77 \%$ de los títulos universitarios, el $62 \%$ de las universidades y el $85 \%$ de las exportaciones de productos manufacturados (INDEC, 2005). Según Feldman (1994), la concentración geográfica de los insumos relacionados con el conocimiento constituye una infraestructura tecnológica que reduce los riesgos y los costos de participar en actividades que suponen una mayor intensidad tecnológica.

\section{Variables explicativas}

Para construir el vector de variables explicativas, se utilizan datos del OEDE y de la Encuesta Nacional de Hogares. Esta distinción es importante porque la información que contiene la base de datos del OEDE se refiere a toda la provincia, mientras que la encuesta se lleva a cabo en relación con muestras de familias de 31 zonas urbanas. Sin embargo, los datos de la Encuesta Nacional de Hogares debían incluirse debido a que no había ninguna fuente de información estadística anual sobre las características demográficas o socioeconómicas de las provincias argentinas (los censos de población, por ejemplo, se hacen cada diez años).

Por lo tanto, se pudieron construir dos tipos de variables: i) variables propias de las regiones, que se relacionan con la evolución de la actividad económica, el mercado de trabajo, el nivel de educación, la estructura industrial y la existencia de economías de aglomeración, y ii) variables propias de los sectores, que permiten tomar en cuenta las condiciones económicas a las que se enfrentan los tres 
grupos de sectores en las distintas regiones, como el crecimiento del mercado, las barreras a la entrada y la salida, la tradición industrial, los efectos de la aglomeración y el precio de los insumos. Como se indica en la sección II, estos factores son muy utilizados en los estudios sobre los países desarrollados. Además, se incluyeron variables ficticias anuales para controlar los factores macroeconómicos ${ }^{8}$.

El próximo paso consistió en introducir variables relacionadas con el nivel de pobreza, la economía informal y la capacidad ociosa, en un intento por captar las singularidades económicas y estructurales de los países en desarrollo. También se incluyó el cuadrado del nivel de pobreza y la informalidad para tener en cuenta los posibles efectos no lineales. El paso final fue examinar si había diferencias entre el centro y la periferia, mediante la inclusión del resultado de multiplicar una variable ficticia que permitía identificar a las provincias más ricas, con las variables que representaban los efectos de la aglomeración y la relación entre las entradas y las salidas.

En los cuadros 3 y 4 se ilustran la definición, las fuentes estadísticas y las estadísticas descriptivas de las variables explicativas. Dichos cuadros también contienen columnas en las que figura el signo que se espera que el coeficiente asociado tenga en relación con la entrada y la salida. Los métodos que se utilizaron para construir esas variables y determinar el signo previsto se explican brevemente más adelante.

\footnotetext{
8 Se dio preferencia a estas en lugar de a las variables macroeconómicas como el crecimiento del PIB debido a los problemas de medición que estas últimas planteaban. La exactitud del crecimiento del PIB en moneda local no se puede asegurar debido a que las cifras oficiales relativas a la inflación no han sido fiables desde 2007. Del mismo modo, el crecimiento del PIB en dólares sería engañoso, debido a la profunda devaluación del peso argentino ocurrida en 2002 (más del 200\%) y la consiguiente apreciación gradual.
} 


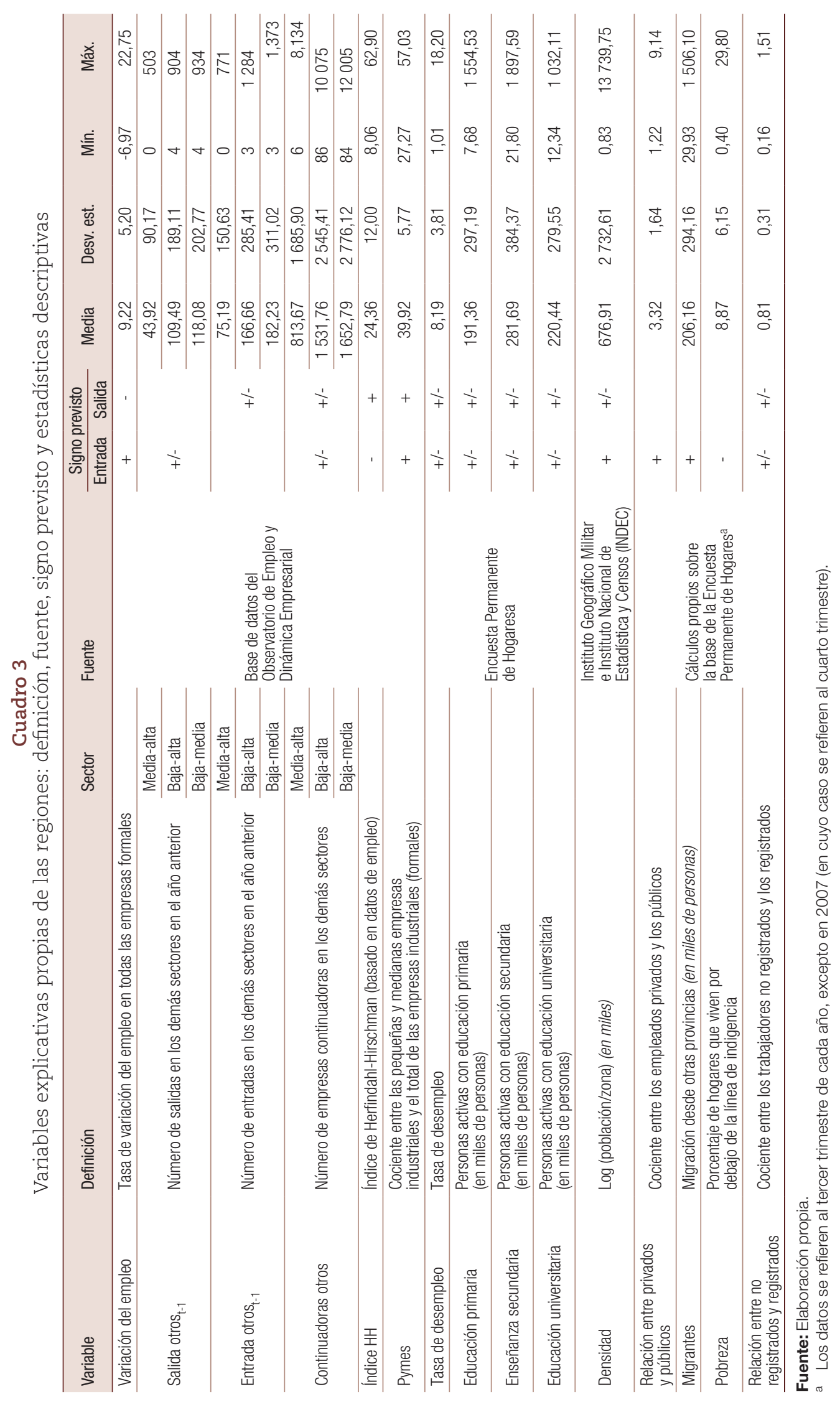




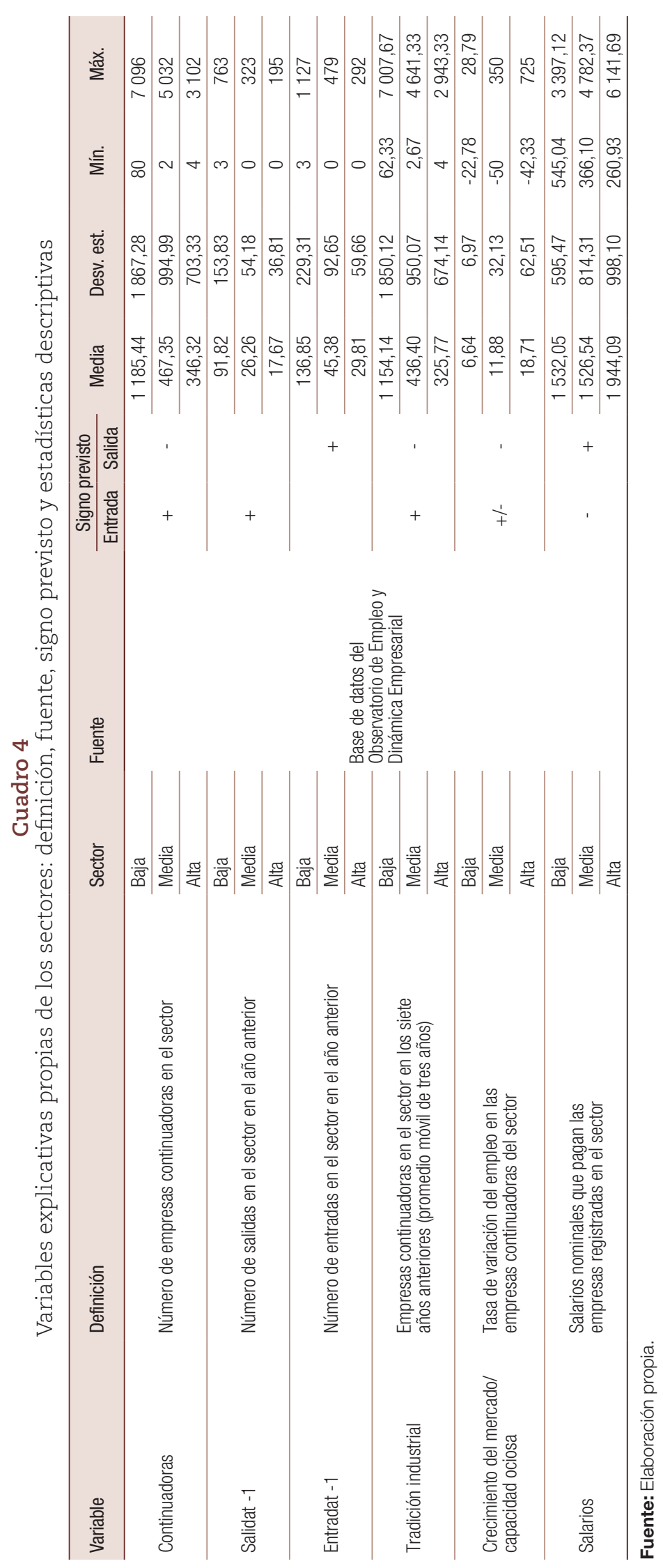




\section{a) Variables propias de las regiones}

Ciclo económico. La tasa de variación del empleo en todas las empresas formales se utiliza como indicador de la evolución de la actividad económica. Se prevé que el coeficiente de esta variable sea positivo en relación con las entradas y negativo en relación con las salidas, lo que refleja el carácter procíclico de ambos procesos. Además, se incluye el número (retardado) de salidas y entradas como otro indicador de la dinámica regional ${ }^{9}$.

Mano de obra. Se utiliza la tasa de desempleo regional para evaluar el efecto del mercado de trabajo en la dinámica empresarial. Como se mencionó anteriormente, es imposible decir a priori cuál será el efecto.

Educación. Se utiliza el número de personas de la población activa que tienen educación primaria, secundaria y universitaria. Se espera que los niveles de educación superiores repercutan más en las actividades de tecnología alta o media.

Concentración espacial. Se utilizan la densidad de la población y su cuadrado como indicadores indirectos de las economías de aglomeración y desaglomeración, respectivamente. Se prevé que el coeficiente de densidad sea positivo en lo que respecta a las entradas, pero tanto positivo como negativo cuando se trata de las salidas. En lo que respecta al coeficiente de densidad al cuadrado, se espera que tenga signo negativo en relación con las entradas y signo positivo con las salidas. También se ha incluido el número de empresas continuadoras como medida adicional de la aglomeración de la actividad económica.

Estructura industrial. El índice de Herfindahl-Hirschman $(H H)$-que mide la ausencia de diversidad - y la proporción de pymes son indicadores indirectos de la estructura industrial de la provincia. Se espera que el índice $\mathrm{HH}$ tenga un efecto negativo sobre la entrada y uno positivo sobre la salida, y que la proporción de pymes tenga un efecto positivo sobre ambas.

Actitudes culturales. En el estudio se incluye el cociente entre los empleados privados y los públicos, así como el número de personas que vienen de otras provincias, para tomar en cuenta las diferencias culturales entre las regiones, que pueden propiciar el surgimiento de empresas. Se espera que ambas variables tengan un efecto positivo en la entrada.

Pobreza. Como indicador de la magnitud de la pobreza se utiliza el porcentaje de hogares que viven por debajo de la línea de indigencia, es decir, el umbral por debajo del cual los ingresos no llegan a costear una canasta básica de alimentos. En 2003, se estimaba que dicho umbral ascendía a unos 38 dólares por adulto. Se espera que, en las regiones más pobres, el número de entradas sea inferior, ya que la proporción de empresarios que cuentan con acceso a los recursos es menor, y la demanda es inferior y menos diversa.

Economía informal. El cociente entre los trabajadores no registrados y los registrados se utiliza como indicador de la estructura productiva regional (por ejemplo, la estacionalidad o la baja productividad de ciertas actividades pueden facilitar el crecimiento del sector informal) o de la falta de controles gubernamentales sobre la economía informal. En el caso de esta variable, es posible que el signo sea positivo o negativo.

\section{b) Variables propias de los sectores}

Entradas y salidas anteriores. Se utiliza el número retardado de entradas (y salidas) en el mismo grupo de sectores para dar cuenta de la interdependencia entre ambos procesos en la ecuación de

\footnotetext{
9 Obsérvese que los efectos de sustitución y desplazamiento se toman en cuenta en relación con las entradas y las salidas en el mismo grupo de sectores.
} 
salida (entrada). Se prevé que las salidas (entradas) anteriores tengan un efecto positivo sobre las entradas (salidas) actuales a causa del efecto de sustitución (desplazamiento). Sin embargo, ese efecto puede verse obstaculizado si los mercados regionales no están saturados y, por consiguiente, la competencia entre las empresas es escasa.

Concentración espacial. El número de empresas continuadoras en cada grupo de sectores se incluye como indicador de los efectos de las economías de localización o del nivel de competencia entre las empresas.

Tradición industrial. Para controlar según las actividades industriales anteriores que se llevaron a cabo en una provincia, se usa el número promedio de empresas continuadoras que había en el mismo sector siete, seis y cinco años antes. Si bien se espera que las empresas continuadoras anteriores alienten la entrada y desalienten la salida, la elevada volatilidad macroeconómica puede mitigar ese efecto.

Salarios. Se utilizan los salarios en cada grupo de sectores para evaluar los efectos del costo de la mano de obra sobre la dinámica empresarial. Dichos salarios corresponden al salario mensual promedio de los trabajadores registrados en el sector privado, y se expresan en términos nominales, habida cuenta de la escasa fiabilidad que las tasas oficiales de inflación tienen en la Argentina desde $2007^{10}$. Se espera que esta variable tenga un efecto negativo sobre las entradas y uno positivo sobre las salidas. No obstante, es posible que sea de poca significación en los países en desarrollo, donde las limitaciones del sistema financiero hacen que muchos empresarios utilicen sus ahorros para obtener el capital inicial necesario (Wang, 2006).

Crecimiento del mercado/capacidad ociosa. En el estudio se utiliza el ritmo de crecimiento del número de empleados en cada grupo de sectores para representar la evolución de la demanda sectorial. Se prevé que el coeficiente de esta variable sea positivo en lo que respecta a las entradas y negativo en relación con las salidas. Sin embargo, el que las empresas continuadoras utilicen su capacidad ociosa puede mitigar ese efecto en el caso de las entradas.

\section{Modelos econométricos}

En el estudio se emplean modelos de datos de panel con variables enteras a fin de calcular las ecuaciones (1) y (2) de la sección III. 111. Los datos de panel permiten controlar algunas características de las provincias (observables o no) que no varían mucho a lo largo del tiempo, como la dotación de recursos naturales, el entorno institucional y la cultura empresarial. Estos datos también ofrecen mayor variabilidad, menos colinealidad entre las variables, más grados de libertad y más eficiencia (Baltagi, 2005).

Se prefirió utilizar modelos de datos de panel en lugar de cálculos de secciones transversales sobre la base de dos pruebas empíricas. En primer lugar, las pruebas del cociente de probabilidad respecto de la varianza de los efectos individuales siempre dan resultados estadísticamente significativos y, por lo tanto, permiten rechazar la validez de los cálculos agrupados (Cameron y Trivedi, 2009). En segundo lugar, se puso a prueba el supuesto de que las observaciones son independientes en los años considerados. Con ese fin, se calculó la matriz de covarianzas en relación con el vector anual de los residuos de Pearson del modelo de regresión de Poisson con datos agrupados (véase más información en Hausman, Hall y Griliches, 1984). El hallazgo de valores grandes en los elementos

\footnotetext{
${ }^{10}$ Los salarios de cada grupo de sectores se construyeron como el promedio ponderado de los salarios nominales de cada sector de dos dígitos, utilizando como índice de ponderación la participación de cada uno de esos sectores en el número total de empresas continuadoras del grupo.

${ }^{11}$ Véanse, por ejemplo, Chappell, Kimenyi y Mayer (1990); Ilmakunnas y Topi (1999); Barbosa, Guimarães y Woodward (2004); Barbosa (2007), y Fritsch y Falck (2007), a fin de hallar ejemplos de aplicaciones en las que se utilizan modelos con variables enteras a fin de explicar la dinámica empresarial en los países desarrollados.
} 
que están fuera de la diagonal de la matriz en todas las especificaciones respalda el supuesto de independencia que sostiene los modelos de datos de panel.

A fin de escoger entre el modelo de Poisson y el binomial negativo, se calculó el cociente entre el estadístico de la calidad del ajuste de Pearson y los grados de libertad de un modelo de Poisson con variables ficticias de las provincias. Como Allison y Waterman (2002) sostienen, si dicho cociente es cercano a uno, no hay pruebas de que los datos presenten demasiada dispersión y los cálculos de Poisson son eficientes. Lamentablemente, los modelos binominales negativos no alcanzaron la convergencia en el modelo de entrada del sector de baja tecnología. Por tanto, en el estudio se presentan los resultados del modelo de Poisson - si bien el cociente, igual a 1,42, es ligeramente superior al valor que proponen Allison y Waterman (2002). En segundo lugar, se llevó a cabo la prueba de Durbin-Hu-Hausman para decidir si se habrían de utilizar efectos fijos o efectos aleatorios. En la mayoría de los modelos no es posible rechazar la hipótesis nula de ausencia de correlación entre las covariables y el efecto individual, lo que significa que el modelo de efectos aleatorios permite obtener estimaciones consistentes. Sin embargo, cuando se rechaza dicha hipótesis, se utilizan modelos de efectos fijos, ya que siempre proporcionan estimaciones consistentes.

\section{Resultados empíricos}

Los cálculos de los modelos con variables enteras se indican en el cuadro 5 , correspondiente a la entrada, y en el cuadro 6, relativo a la salida. En las columnas [1] se presentan los resultados de la especificación que contiene variables que suelen utilizarse en los estudios sobre los países desarrollados, y en las columnas [2] se incluyen variables que reflejan las características propias de los países en desarrollo (la pobreza, el tamaño del sector informal y la capacidad ociosa), así como el patrón centro-periferia que se halla en la Argentina (Calá, Arauzo-Carod y Manjón-Antolín, 2015; Calá, Manjón-Antolín y Arauzo-Carod, 2016).

Tras analizar los resultados relativos a la entrada de empresas (véanse las columnas [2] del cuadro 5), es evidente que los relacionados con las actividades de baja tecnología, que representan aproximadamente el 65\% del total de las entradas durante el período que se examina, coinciden en gran medida con los que se hallaron en estudios anteriores sobre el sector manufacturero en su conjunto (Calá, Manjón-Antolín y Arauzo-Carod, 2016). Sin embargo, el efecto de las covariables no es homogéneo en todos los sectores. Por ejemplo, la evolución de la demanda regional solo tiene relevancia en relación con las actividades de baja tecnología, mientras que las variables estructurales (como la proporción de pymes o la diversificación industrial), los factores culturales (como el cociente entre los empleados privados y los públicos o la proporción de migrantes) o el nivel salarial, solo son relevantes en el caso de los sectores de tecnología media y alta. Esto sugiere que los entornos económicos favorables fomentan la creación de empresas solo en los sectores tradicionales, pero no son suficientes para promover cambios estructurales regionales impulsados por la entrada de empresas en los sectores de tecnología media y alta. 


\section{Cuadro 5}

Argentina: factores que determinan la entrada de empresas por grupo de sectores, 2003-2008

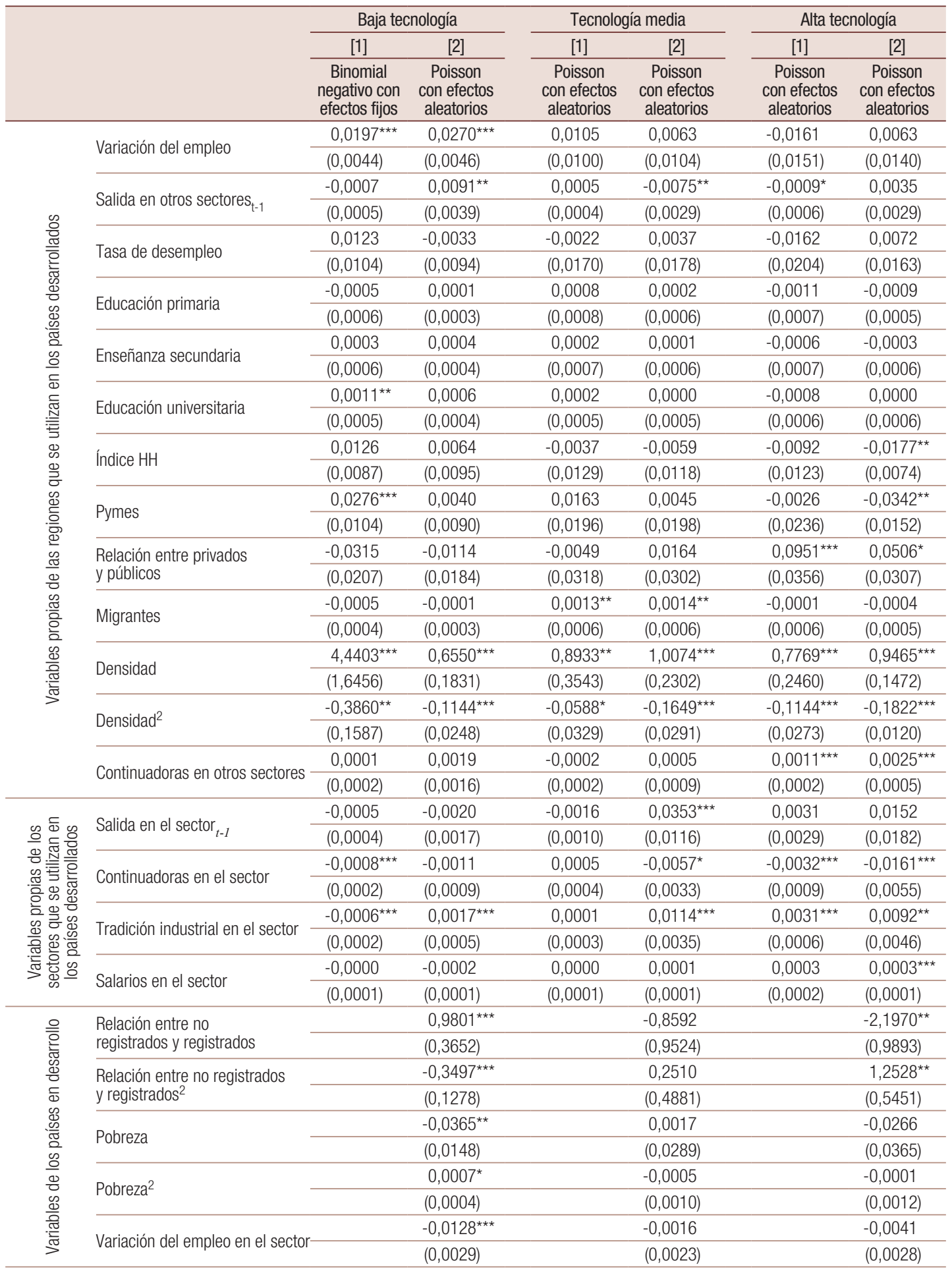


Cuadro 5 (conclusión)

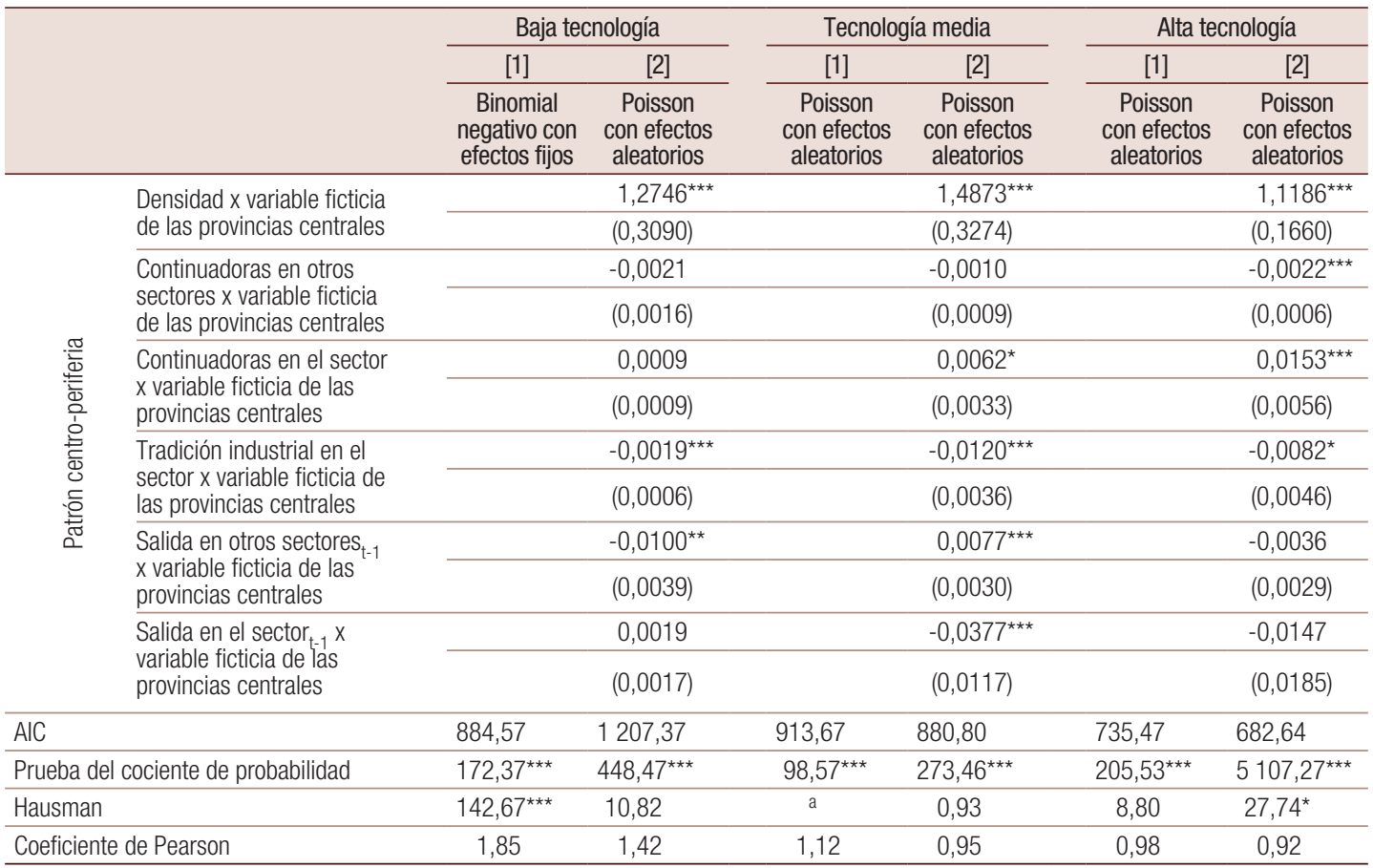

Fuente: Elaboración propia.

Nota: El número de observaciones es de 144. En los sectores de alta tecnología, el número de observaciones es de 138 en los modelos de efectos fijos. Errores estándar entre paréntesis. Los asteriscos indican la significación estadística del coeficiente: ** valor de $p<0,01,{ }^{*}$ * valor de $p<0,05$, * valor de $p<0,1$. Se incluyen variables ficticias anuales en todas las especificaciones.

a Se halló un estadístico negativo que no se informa.

\section{Cuadro 6}

Argentina: factores que determinan la salida de empresas por grupo de sectores, 2003-2008

\begin{tabular}{|c|c|c|c|c|c|c|c|}
\hline & & \multicolumn{2}{|c|}{ Baja tecnología } & \multicolumn{2}{|c|}{ Tecnología media } & \multicolumn{2}{|c|}{ Alta tecnología } \\
\hline & & [1] & [2] & [1] & [2] & [1] & [2] \\
\hline & & $\begin{array}{l}\text { Poisson } \\
\text { con efectos } \\
\text { aleatorios }\end{array}$ & $\begin{array}{c}\text { Poisson } \\
\text { con efectos } \\
\text { aleatorios }\end{array}$ & $\begin{array}{l}\text { Poisson con } \\
\text { efectos fijos }\end{array}$ & $\begin{array}{l}\text { Poisson con } \\
\text { efectos fijos }\end{array}$ & $\begin{array}{c}\text { Poisson } \\
\text { con efectos } \\
\text { aleatorios }\end{array}$ & $\begin{array}{c}\text { Poisson } \\
\text { con efectos } \\
\text { aleatorios }\end{array}$ \\
\hline \multirow{22}{*}{ 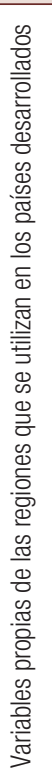 } & \multirow{2}{*}{ Variación del empleo } & $-0,0084$ & 0,0003 & $-0,0083$ & $-0,0052$ & $-0,0135$ & 0,0063 \\
\hline & & $(0,0053)$ & $(0,0058)$ & $(0,0145)$ & $(0,0154)$ & $(0,0179)$ & $(0,0168)$ \\
\hline & \multirow{2}{*}{ Tasa de desempleo } & $-0,0172^{*}$ & $-0,0034$ & $-0,0247$ & $-0,0187$ & 0,0260 & 0,0300 \\
\hline & & $(0,0098)$ & $(0,0102)$ & $(0,0251)$ & $(0,0283)$ & $(0,0255)$ & $(0,0212)$ \\
\hline & \multirow{2}{*}{ Educación primaria } & $-0,0006^{\star *}$ & $-0,0004$ & $0,0020^{*}$ & 0,0015 & $-0,0016^{\star *}$ & $-0,0015^{\star \star *}$ \\
\hline & & $(0,0003)$ & $(0,0003)$ & $(0,0012)$ & $(0,0014)$ & $(0,0007)$ & $(0,0006)$ \\
\hline & \multirow{2}{*}{ Enseñanza secundaria } & $-0,0000$ & $0,0009^{\star \star}$ & $-0,0003$ & $-0,0001$ & $-0,0008$ & 0,0007 \\
\hline & & $(0,0005)$ & $(0,0005)$ & $(0,0013)$ & $(0,0014)$ & $(0,0010)$ & $(0,0007)$ \\
\hline & \multirow{2}{*}{ Educación universitaria } & $0,0007^{*}$ & $0,0007^{*}$ & $-0,0006$ & $-0,0003$ & 0,0011 & $0,0013^{\star}$ \\
\hline & & $(0,0004)$ & $(0,0004)$ & $(0,0010)$ & $(0,0010)$ & $(0,0007)$ & $(0,0006)$ \\
\hline & \multirow{2}{*}{ Índice HH } & $-0,0030$ & $-0,0112^{\star \star}$ & 0,0202 & $-0,0033$ & $-0,0027$ & $-0,0284^{\star \star \star}$ \\
\hline & & $(0,0066)$ & $(0,0055)$ & $(0,0247)$ & $(0,0406)$ & $(0,0128)$ & $(0,0099)$ \\
\hline & \multirow{2}{*}{ Pymes } & $-0,0070$ & $-0,0072$ & $0,0818^{\star \star \star}$ & $0,0923^{\star \star \star}$ & 0,0280 & 0,0036 \\
\hline & & $(0,0098)$ & $(0,0090)$ & $(0,0292)$ & $(0,0347)$ & $(0,0224)$ & $(0,0180)$ \\
\hline & \multirow{2}{*}{ Entrada en otros sectores ${ }_{\mathrm{t}-1}$} & 0,0002 & $-0,0111^{\star \star \star}$ & 0,0004 & 0,0014 & $-0,0006$ & 0,0009 \\
\hline & & $(0,0003)$ & $(0,0039)$ & $(0,0006)$ & $(0,0039)$ & $(0,0005)$ & $(0,0040)$ \\
\hline & \multirow{2}{*}{ Densidad } & $0,5842^{\star \star \star}$ & $0,5945^{\star \star \star}$ & $-2,6954$ & $-7,1567$ & $0,5644^{\star *}$ & $0,3969^{\star *}$ \\
\hline & & $(0,1387)$ & $(0,1175)$ & $(7,1660)$ & $(8,2045)$ & $(0,2605)$ & $(0,1979)$ \\
\hline & \multirow{2}{*}{ Densidad $^{2}$} & $-0,0727^{\star \star \star}$ & $-0,1018^{\star \star \star}$ & 0,8084 & 0,5324 & $-0,0945^{\star \star \star}$ & $-0,1346^{\star \star *}$ \\
\hline & & $(0,0160)$ & $(0,0149)$ & $(0,7046)$ & $(1,0326)$ & $(0,0311)$ & $(0,0148)$ \\
\hline & \multirow{2}{*}{$\begin{array}{l}\text { Continuadoras en } \\
\text { otros sectores }\end{array}$} & $-0,0001$ & 0,0007 & $-0,0006$ & 0,0035 & $0,0011^{\star \star \star}$ & $0,0029^{\star \star \star}$ \\
\hline & & $(0,0002)$ & $(0,0013)$ & $(0,0007)$ & $(0,0041)$ & $(0,0003)$ & $(0,0008)$ \\
\hline
\end{tabular}


Cuadro 6 (conclusión)

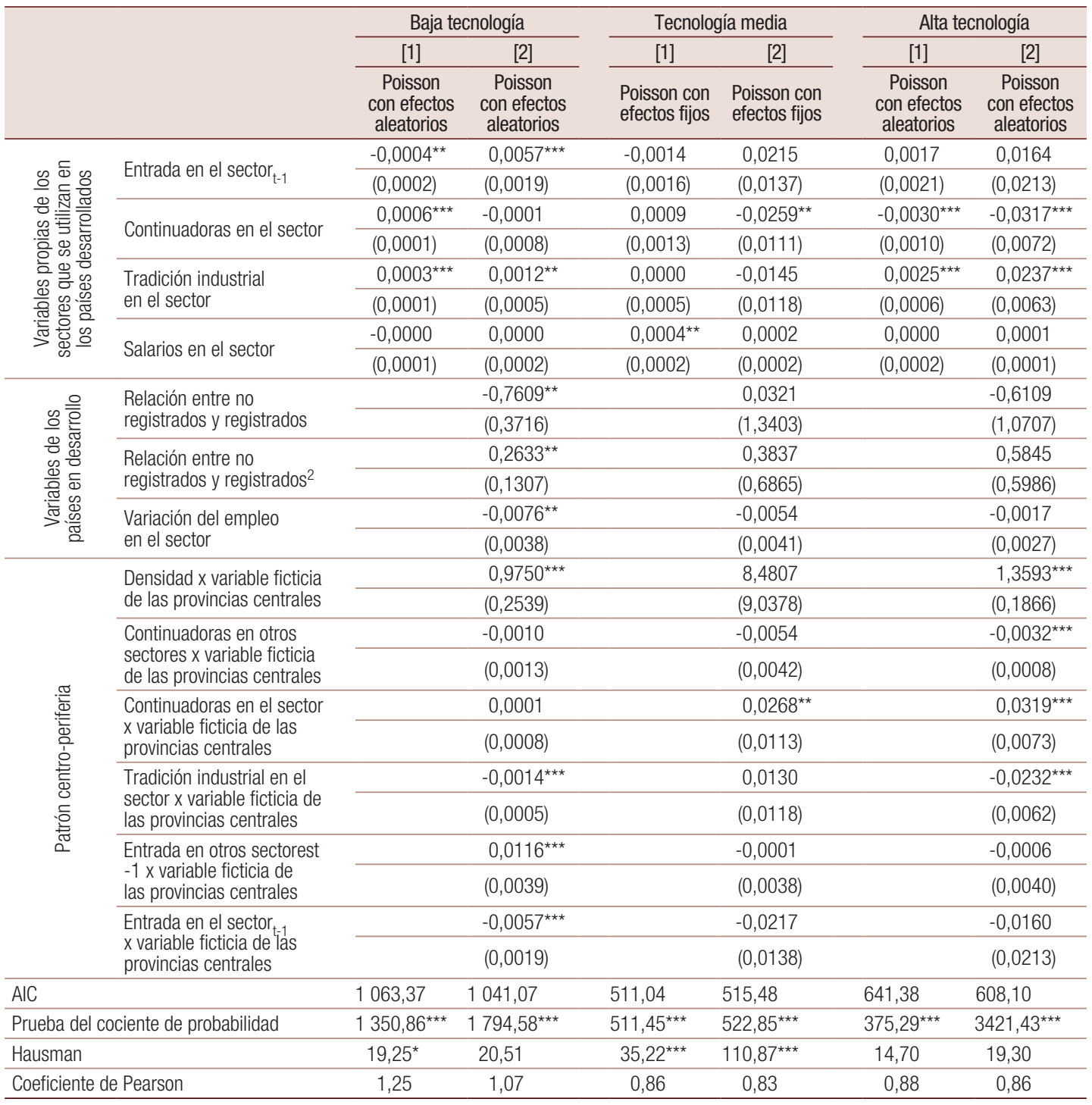

Fuente: Elaboración propia.

Nota: El número de observaciones es de 144. Errores estándar entre paréntesis. Los asteriscos indican la significación estadística del coeficiente: * * valor de $p<0,01,{ }^{*}$ * valor de $p<0,05$, * valor de $p<0,1$. Se incluyen variables ficticias anuales en todas las especificaciones.

También hay efectos importantes de aglomeración y dispersión producidos por la densidad de población. Los primeros son grandes sobre todo en las regiones centrales, donde la mayor densidad de población fomenta la entrada incluso más que en la periferia. En las provincias centrales, las economías de aglomeración de los sectores de tecnología media y alta también son impulsadas por el número de empresas continuadoras en el mismo sector (economías de localización). Es interesante señalar que los efectos de la aglomeración sobre las actividades de alta tecnología también proceden de la concentración de empresas en otros sectores, lo que es congruente con la idea de las economías de urbanización, como en los países desarrollados (Henderson, Kuncoro y Turner, 1995). Esta conclusión pone de manifiesto la importancia de la diversificación regional en lo que respecta a la promoción de nuevas empresas de alta tecnología, y está en consonancia con el impacto negativo de la concentración industrial sobre las entradas en el sector de alta tecnología. 
Las variables que representan las particularidades de la Argentina como país en desarrollo son muy significativas, en particular en los sectores de baja tecnología. Por ejemplo, el signo negativo de la variable correspondiente a la pobreza está en consonancia con el menor poder adquisitivo y la menor cantidad de recursos financieros, humanos y de capital social que los empresarios de las zonas pobres tienen a disposición. Además, el efecto positivo del cuadrado de esa variable sugiere que los niveles elevados de pobreza estimulan la creación de empresas (posiblemente pequeñas) con pocas barreras a la entrada. El impacto de la economía informal tampoco es lineal. El que la economía informal sea pequeña alienta la entrada al incitar a las personas a tener iniciativa empresarial o al brindar la posibilidad de subcontratar actividades. Sin embargo, cuando el sector informal crece demasiado, la competencia de las empresas formales con las informales puede obstaculizar la entrada de las primeras ${ }^{12}$. En particular, esta variable tiene efectos opuestos sobre la entrada en lo que respecta a las actividades de alta y baja tecnología. Además, a medida que el empleo en el sector aumenta, cabe esperar que el número de entradas en el sector de baja tecnología sea inferior, lo que sugiere que el aumento de la demanda sectorial se satisface utilizando la capacidad ociosa y no creando empresas nuevas.

Por último, muchas variables, en particular los efectos de sustitución y las economías de aglomeración pasadas y actuales, muestran efectos opuestos en el centro y la periferia, detalle que se pasa por alto en las especificaciones en las que no se distingue entre ambos grupos de provincias y, por lo tanto, se superponen los efectos positivos y negativos. Las conclusiones indican que promover la entrada de empresas de alta tecnología puede ser especialmente difícil en las regiones rezagadas, porque no suele haber una cantidad suficiente de empresas conexas que permita crear las condiciones necesarias para el surgimiento de economías externas de escala.

En el cuadro 6 figuran resultados análogos relativos a la salida de empresas. Una vez más, los resultados relacionados con la salida de empresas en actividades de baja tecnología (que representan el $68 \%$ del total de las salidas) coinciden con los que se obtuvieron en estudios anteriores que se centraban en las salidas globales (Calá, Arauzo-Carod y Manjón-Antolín, 2015). En particular, las entradas anteriores en el sector producen un efecto de sustitución en las regiones periféricas, pero evitan las salidas en el centro. Esto sugiere que el efecto de la puerta giratoria es más intenso en las regiones más pobres y que tal vez la supervivencia (presumiblemente) más breve esté relacionada con el tamaño pequeño del mercado en esas regiones rezagadas. Lo anterior siembra dudas sobre la utilidad de las políticas de promoción de la entrada en esas regiones, que, en última instancia, quizás solo causen más salidas. La tradición industrial y las entradas en otros sectores también tienen efectos opuestos en las provincias centrales y en las periféricas. En particular, la repercusión negativa de las entradas anteriores que se observa en la periferia puede ser un indicador de las perspectivas de la actividad manufacturera en la región. Además, los resultados indican que el grado de concentración industrial y el crecimiento del mercado tienen un efecto negativo sobre la salida, mientras que el nivel educativo de la fuerza de trabajo tiene un efecto positivo. Esto puede estar relacionado con la mayor competencia en las zonas donde el capital humano tiene un nivel superior.

En las zonas densas, hay empresas que se ven forzadas a salir del mercado, aunque este efecto se invierte en las zonas donde la densidad es muy alta. Este resultado puede atribuirse a los efectos de la competencia y a las diferencias dentro de cada provincia, por ejemplo, entre las zonas densas que se especializan en la prestación de servicios (sobre todo servicios públicos) y las regiones industriales menos pobladas, donde es más fácil preservar la actividad manufacturera. En cualquier caso, se trata de un tema digno de investigar en el futuro. El efecto de la dispersión, en particular, es más pronunciado en las regiones centrales, donde la densidad de la población fomenta la salida aún más que en el resto del país.

\footnotetext{
${ }^{12}$ El que el sector informal sea más grande también puede ser reflejo de la ausencia de controles gubernamentales en algunas provincias, lo que puede desalentar a los empresarios de crear empresas formales.
} 
Por último, el efecto de la economía informal en el sector de baja tecnología es muy similar al que se observa en el conjunto de la industria manufacturera (Calá, Arauzo-Carod y Manjón-Antolín, 2015). Si bien las economías informales de pequeño tamaño evitan las salidas, el efecto es el contrario cuando dichas economías crecen más allá de determinado nivel. El efecto negativo inicial sobre la salida puede estar relacionado con los menores costos inherentes del sector informal, o la mayor flexibilidad presente a la hora de contratar personal, en tanto que cabe esperar un efecto positivo cuando las empresas formales tienen que competir con las informales por los recursos o el acceso a los mercados. Sorprendentemente, al parecer los vínculos entre el sector formal y el informal solo son pertinentes en los sectores de baja tecnología.

Al igual que ocurre con el proceso de entrada, en los sectores de tecnología media las salidas son mucho menos sistemáticas que en los demás sectores ${ }^{13}$. La proporción de pymes tiene un efecto positivo, lo que refleja la "desventaja de la pequeñez" (Strotmann, 2007). También existe un efecto de competencia que las empresas continuadoras ejercen en las zonas centrales, mientras que, en la periferia, donde las economías de localización parecen ser más importantes, se observa el efecto opuesto.

Las salidas en los sectores de alta tecnología están impulsadas en gran medida por las deseconomías de aglomeración que surgen de la densidad de población y del número de empresas continuadoras en otros sectores. Sin embargo, hay diferencias notables entre el centro y la periferia. En las zonas centrales, las empresas continuadoras del mismo sector empujan a las empresas hacia fuera del mercado (efecto de competencia), mientras que, en las provincias periféricas, dichas empresas inducen economías de localización que evitan la salida. Al mismo tiempo, las empresas continuadoras de otros sectores retienen a las empresas en las provincias centrales (que es el resultado esperado cuando la estructura industrial es densa o cuando hay economías de urbanización), pero promueven la salida en las regiones periféricas. La tradición industrial en el sector también es pertinente y tiene el efecto opuesto en las regiones centrales (negativo) y en la periferia (positivo). Estos resultados indican que algunos estudios de caso sobre empresas de alta tecnología en la región central y en la periférica pueden arrojar luz sobre las deficiencias que esas empresas enfrentan en ambos grupos de provincias.

\section{Conclusión}

En el presente estudio se analizan los factores que determinan la entrada y la salida de las empresas en los países en desarrollo, y se utiliza la Argentina como caso ilustrativo. Este es, a nuestro entender, el primer intento de explicar la dinámica empresarial regional en una economía en desarrollo, mediante el uso de variables regionales y sectoriales.

Los resultados sugieren que, para explicar la dinámica empresarial en esos países, se deben tener en cuenta varias particularidades de las economías en desarrollo. En la Argentina, por ejemplo, los resultados revelan un efecto de sustitución entre el uso de la capacidad ociosa y la creación de nuevas empresas, así como un efecto no lineal del nivel de pobreza sobre las entradas. Esto último significa que las políticas que solo tienen por objeto promover la creación de nuevas empresas pueden tener un efecto limitado, ya que para reducir las tasas de pobreza probablemente se requieran medidas a largo plazo. Además, los resultados indican que el tamaño del sector informal tiene un efecto no lineal en la entrada y la salida, y que dicho efecto difiere entre los sectores de alta y baja tecnología. Esto sugiere que los vínculos entre el sector formal y el informal pueden ser sumamente complejos y que se requieren más investigaciones para comprender esa estrecha relación.

\footnotetext{
${ }^{13}$ El hecho de que las actividades de tecnología media probablemente compartan determinadas características con los sectores de tecnología baja y con los de tecnología alta hace que resulte más difícil hallar los factores que determinan la dinámica empresarial en el grupo de sectores correspondiente a dichas actividades.
} 
El estudio también muestra que los factores que explican la entrada y la salida en los sectores de alta y baja tecnología son diferentes. En particular, las variables propias de los países en desarrollo repercuten sobre todo en las entradas y las salidas de los sectores de baja tecnología. Esto sugiere que, posiblemente, las medidas que se adopten en materia de políticas a fin de fomentar la creación de empresas y evitar su salida solo lograrán su cometido si en ellas se toma en cuenta la mezcla industrial de cada región geográfica.

Por último, hay datos que indican la existencia de un patrón de centro-periferia según el cual muchas variables tienen efectos opuestos según se trate de las provincias centrales o las rezagadas. Esto significa que, si no se hace una distinción entre las regiones centrales y las periféricas, no es posible evaluar de forma adecuada los efectos geográficos sobre la dinámica empresarial. Por consiguiente, las políticas destinadas a promover la iniciativa empresarial en todo el país no deberían basarse en los resultados y las experiencias extraídas únicamente de las regiones centrales.

En futuras ampliaciones del presente estudio se debería evaluar la similitud de los efectos de las variables explicativas en las empresas manufactureras que no son locales y en el sector de los servicios. En los estudios posteriores también se deberían analizar los efectos de la dinámica empresarial regional sobre algunos indicadores del desempeño económico, como la creación de empleo y la innovación regional.

\section{Bibliografía}

Agarwal, R. y M. Gort (1996), "The evolution of markets and entry, exit and survival of firms", The Review of Economics and Statistics, vol. 78, N ${ }^{\circ} 3$, Cambridge, Massachusetts, The MIT Press.

Allison, P. D. y R. P. Waterman (2002), "Fixed-effects negative binomial regression models", Sociological Methodology, vol. 32, No 1, Wiley.

Álvarez, R. y S. Vergara (2013), "Trade exposure, survival and growth of small and medium-size firms", International Review of Economics \& Finance, vol. 25, Amsterdam, Elsevier.

(2010), "Exit in developing countries: economic reforms and plant heterogeneity", Economic Development and Cultural Change, vol. 58, N ${ }^{\circ}$ 3, Chicago, The University of Chicago Press.

Arauzo-Carod, J. M. y otros (2007), "Regional and sector-specific determinants of industry dynamics and the displacement-replacement effects", Empirica, vol. 34, № 2, Springer.

Armington, C. y Z. J. Acs (2002), "The determinants of regional variation in new firm formation", Regional Studies, vol. 36, № 1, Taylor \& Francis.

Audretsch, D. B. (1995a), "Innovation, growth and survival", International Journal of Industrial Organization, vol. 13, № 4, Amsterdam, Elsevier.

_ (1995b), Innovation and Industry Evolution, Cambridge, Massachusetts, The MIT Press.

Audretsch, D. B. y M. Fritsch (1999), "The industry component of regional new firm formation processes", Review of Industrial Organization, vol. 15, № 3, Springer.

Audretsch, D. B. y M. Keilbach (2005), "Entrepreneurship capital and regional growth", The Annals of Regional Science, vol. 39, № 3, Springer.

Baltagi, B. H. (2005), Econometric Analysis of Panel Data, Chichester, Wiley.

Barbosa, N. (2007), "An integrative model of firms' entry decisions", Applied Economics Quarterly, vol. 53, $N^{\circ} 1$, Berlín, Duncker \& Humblot.

Barbosa, N., P. Guimarães y D. Woodward (2004), "Foreign firm entry in an open economy: the case of Portugal", Applied Economics, vol. 36, № 5, Taylor \& Francis.

Bennett, J. (2010), "Informal firms in developing countries: entrepreneurial stepping stone or consolation prize?", Small Business Economics, vol. 34, № 1, Springer.

Bresnahan, T. F. y P. C. Reiss (1991), "Entry and competition in concentrated markets", Journal of Political Economy, vol. 99, № 5, Chicago, The University of Chicago Press.

Brixy, U. y R. Grotz (2007), "Regional patterns and determinants of birth and survival of new firms in Western Germany”, Entrepreneurship \& Regional Development, vol. 19, № 4, Taylor \& Francis. 
Bruton, G. D., D. Ahlstrom y K. Obloj (2008), "Entrepreneurship in emerging economies: where are we today and where should the research go in the future", Entrepreneurship Theory and Practice, vol. 32, № 1, Wiley.

Buss, T. F. y X. Lin (1990), "Business survival in rural America: a three-state study", Growth and Change, vol. 21, No 3 , Wiley.

Calá, C. D., M. Manjón-Antolín y J. M. Arauzo-Carod (2016), "Regional determinants of firm entry in a developing country", Papers in Regional Science, vol. 95, N², Wiley.

(2015), "The determinants of exit in a developing country: core and peripheral regions", The Annals of Regional Science, vol. 54, N³, Springer.

Cameron, A. C. y P. K. Trivedi (2009), Microeconometrics Using Stata, Texas, Stata Press.

Carree, M., S. Santarelli e I. Verheul (2008), "Firm entry and exit in Italian provinces and the relationship with unemployment", International Entrepreneurship Management Journal, vol. 4, № 2, Springer.

Carree, M. y R. Thurik (1996), "Entry and exit in retailing: incentives, barriers, displacement and replacement", Review of Industrial Organization, vol. 11, N², Springer.

Carree, M., I. Verheul y E. Santarelli (2011), "Sectoral patterns of firm exit in Italian provinces", Journal of Evolutionary Economics, vol. 21, N 3, Springer.

Cassiolato, J. E., C. B. Pagola y H. M. Lastres (2009), "Technical change and structural inequalities: converging approaches to problems of underdevelopment", Techno-Economic Paradigms: Essays in Honour of Carlota Perez, W. J. Drechsler, R. Kattel y E. S. Reinert (eds.), Londres, Anthem Press.

CEPAL (Comisión Económica para América Latina y el Caribe) (2007), "Progreso técnico y cambio estructural en América Latina y el Caribe", Documentos de Proyectos (LC/W.136), Santiago.

Chappell, W., M. Kimenyi y W. Mayer (1990), "A poisson probability model of entry and market structure with an application to U.S. industries during 1972-77", Southern Economic Journal, vol. 56, № 4, Chattanooga, Tennessee, Southern Economic Association.

Eslava, M. y otros (2006), "Plant turnover and structural reforms in Colombia", IMF Staff Papers, N 53, número especial, Washington, D.C., Fondo Monetario Internacional (FMI).

Feldman, M. P. (1994), The Geography of Innovation, vol. 2, Springer.

Forsyth, G. D. (2005), "A note on small business survival rates in rural areas: the case of Washington state", Growth and Change, vol. 36, N³, Wiley.

Fotopoulos, G. y N. Spence (1998), "Accounting for net entry into Greek manufacturing by establishments of varying size", Small Business Economics, vol. 11, N², Springer.

Frazer, G. (2005), "Which firms die? A look at manufacturing firm exit in Ghana", Economic Development and Cultural Change, vol. 53, N³, Chicago, The University of Chicago Press.

Fritsch, M. y O. Falck (2007), "New business formation by industry over space and time: a multidimensional analysis", Regional Studies, vol. 41, N², Taylor \& Francis.

Fritsch, M., U. Brixy y O. Falck (2006), "The effect of industry, region, and time on new business survival. A multi-dimensional analysis", Review of Industrial Organization, vol. 28, N³, Springer.

Ghani, E., W. Kerr y S. O'Connell (2014), "Spatial determinants of entrepreneurship in India", Regional Studies, vol. 48, N 6, Taylor \& Francis.

Günalp, B. y S. M. Cilasun (2006), "Determinants of entry in Turkish manufacturing industries", Small Business Economics, vol. 27, N²-3, Springer.

Hausman, J., B. H. Hall y Z. Griliches (1984), "Econometric models for count data with an application to the patents-R \& D relationship", Econometrica, vol. 52, № 4, Nueva York, The Econometric Society.

Henderson, V., A. Kuncoro y M. Turner (1995), "Industrial development in cities", Journal of Political Economy, vol. 103, N5, Chicago, The University of Chicago Press.

Huiban, J. P. (2011), "The spatial demography of new plants: urban creation and rural survival”, Small Business Economics, vol. 37, N 1, Springer.

Ilmakunnas, P. y J. Topi (1999), "Microeconomic and macroeconomic influences on entry and exit of firms", Review of Industrial Organization, vol. 15, N³, Springer.

INDEC (Instituto Nacional de Estadística y Censos) (2005), Anuario Estadístico de la República Argentina, Buenos Aires.

Kantis, H., P. Angelelli y V. Moori Koening (2005), Developing Entrepreneurship: Experience in Latin America and Worldwide, Washington, D.C., Banco Interamericano de Desarrollo (BID).

Katz, J. y G. Bernat (2011), "Exit-entry, productivity growth and structural change in response to changes in macroeconomic policy: evidence from Argentina", Innovation and Development, vol. 1, $\mathrm{N}^{\circ} 2$, Taylor \& Francis. 
Katz, J. y G. Stumpo (2001), "Regímenes sectoriales, productividad y competitividad internacional", Revista de la CEPAL, № 75 (LC/G.2150-P), Santiago, Comisión Económica para América Latina y el Caribe (CEPAL).

Keeble, D. y S. Walker (1994), "New firms, small firms and dead firms: spatial patterns and determinants in the United Kingdom", Regional Studies, vol. 28, N 4, Taylor \& Francis.

Kosacoff, B. y A. Ramos (1999), "El debate sobre política industrial", Revista de la CEPAL, № 68 (LC/G.2039-P), Santiago, Comisión Económica para América Latina y el Caribe (CEPAL).

Lay, T. J. (2003), "The determinants of and interaction between entry and exit in Taiwan's manufacturing", Small Business Economics, vol. 20, № 4, Springer.

Littunen, H., E. Storhammar y T. Nenonen (1998), "The survival of firms over the critical first 3 years and the local environment", Entrepreneurship \& Regional Development vol. 10, № 3, Taylor \& Francis.

López, R. A. (2006), "Imports of intermediate inputs and plant survival", Economics Letters, vol. 92, № 1, Amsterdam, Elsevier.

MTEySS (Ministerio de Trabajo, Empleo y Seguridad Social) (2007), "La creación de empresas durante la etapa post convertibilidad (2003-2005): impacto sobre el empleo asalariado registrado", Trabajo, ocupación y empleo. Salarios, empresas y empleo 2003-2006, serie Estudios, № 5, Buenos Aires.

Naudé, W. y otros (2008), "Regional determinants of entrepreneurial start-ups in a developing country", Entrepreneurship \& Regional Development: An International Journal, vol. 20, № 2, Taylor \& Francis.

Nurmi, S. (2006), "Sectoral differences in plant start-up size in the Finnish economy", Small Business Economics, vol. 26, $\mathrm{N}^{\circ} 1$, Springer.

Nyström, K. (2007), "An industry disaggregated analysis of the determinants of regional entry and exit", Annals of Regional Science, vol. 41, № 4, Springer.

Ocampo, J. A., C. Rada y L. Taylor (2009), Growth and Policy in Developing Countries: A Structuralist Approach, Nueva York, Columbia, University Press.

Ozturk, S. y C. Kilic (2012), "Patterns and determinants of entry and exit in Turkish manufacturing industries", International Journal of Arts and Commerce, vol. 1, № 5 [en línea] http://ijac.org.uk/images/frontlmages/ gallery/Nol._1_No._5/11.pdf.

Reynolds, P., D. J. Storey y P. Westhead (1994), "Cross-national comparisons of the variation in new firm formation rates", Regional Studies, vol. 28, No 4, Taylor \& Francis.

Rocha, H. O. y R. Sternberg (2005), "Entrepreneurship: the role of clusters theoretical perspectives and empirical evidence from Germany", Small Business Economics, vol. 24, №3, Springer.

Santarelli, E., M. Carree e I. Verheul (2009), "Unemployment and firm entry and exit: an update on a controversial relationship", Regional Studies, vol. 43, № 8, Taylor \& Francis.

Santarelli, E. y H. T. Tran (2012), "Growth of incumbent firms and entrepreneurship in Vietnam", Growth and Change, vol. 43, № 4, Wiley.

Schneider, F. (2005), "Shadow economies around the world: what do we really know?", European Journal of Political Economy, vol. 21, № 3, Amsterdam, Elsevier.

Scott, A. J. y M. Storper (2007), "Regions, globalization, development", Regional Studies, vol. 41(S1), Taylor \& Francis.

Shapero, A. (1983), New Business Formation, Enschede.

Spilling, O. R. (1996), "Regional variation of new firm formation: the Norwegian case", Entrepreneurship and Regional Development, vol. 8, № 3, Taylor \& Francis.

Stiglitz, J. (1998), "Towards a new paradigm for development: strategies, policies, and processes" [en línea] http://citeseerx.ist.psu.edu/viewdoc/download?doi=10.1.1.199.9708\&rep=rep1\&type=pdf.

Strotmann, H. (2007), "Entrepreneurial survival”, Small Business Economics, vol. 28, № 1, Springer.

Sunkel, O. (1978), "La dependencia y la heterogeneidad estructural”, Trimestre Económico, vol. 45, № 177(1), Ciudad de México, Fondo de Cultura Económica.

Vernon, R. (1966), "International investment and international trade in the product cycle", The Quarterly Journal of Economics, vol. 80, № 2, Oxford, Oxford University Press.

Wang, S. (2006), "Determinants of new firm formation in Taiwan", Small Business Economics, vol. 27, $N^{\circ} 4-5$, Springer.

Wilson, D. y R. Purushothaman (2006), "Dreaming with BRICs: the path to 2050", Emerging Economies and the Transformation of International Business. Brazil, Russia, India and China, S. Jain (ed.), Cheltenham, Edward Elgar Publishing Ltd. 


\section{Anexo A1}

Cuadro A1.1

Argentina: clasificación industrial, 2008

(En porcentajes)

\begin{tabular}{|c|c|c|c|c|}
\hline Grupo & Código & Sector & Empresas & Empleados \\
\hline \multirow{10}{*}{$\begin{array}{l}\text { Baja } \\
\text { tecnología }\end{array}$} & 15 & Elaboración de productos alimenticios y bebidas & 22,6 & 26,6 \\
\hline & 16 & Elaboración de productos de tabaco & 0,0 & 0,4 \\
\hline & 17 & Fabricación de productos textiles & 4,9 & 5,5 \\
\hline & 18 & Fabricación de prendas de vestir; adobo y teñido de pieles & 6,8 & 4,5 \\
\hline & 19 & $\begin{array}{l}\text { Curtido y adobo de cueros; fabricación de maletas, bolsos de } \\
\text { mano, artículos de talabartería y guarnicionería, y calzado }\end{array}$ & 3,0 & 3,3 \\
\hline & 20 & $\begin{array}{l}\text { Producción de madera y fabricación de productos de madera y corcho, excepto } \\
\text { muebles; fabricación de artículos de paja y de materiales trenzables }\end{array}$ & 5,9 & 3,2 \\
\hline & 22 & Actividades de edición e impresión y de reproducción de grabaciones & 6,9 & 4,8 \\
\hline & 26 & Fabricación de otros productos minerales no metálicos & 3,3 & 3,4 \\
\hline & 36 & Fabricación de muebles; industrias manufactureras N. C. P. & 5,7 & 3,4 \\
\hline & 37 & Reciclamiento & 0,3 & 0,3 \\
\hline \multicolumn{3}{|c|}{ Total baja tecnología } & 59,4 & 55,4 \\
\hline \multirow{5}{*}{$\begin{array}{l}\text { Tecnología } \\
\text { media }\end{array}$} & 21 & Fabricación de papel y de productos de papel & 1,5 & 2,5 \\
\hline & 23 & Fabricación de coque, productos de la refinación del petróleo y combustible nuclear & 0,1 & 0,5 \\
\hline & 25 & Fabricación de productos de caucho y plástico & 5,3 & 5,4 \\
\hline & 27 & Fabricación de metales comunes & 2,1 & 3,5 \\
\hline & 28 & Fabricación de productos elaborados de metal, excepto maquinaria y equipo & 14,4 & 8,7 \\
\hline \multicolumn{3}{|c|}{ Total tecnología media } & 23,4 & 20,6 \\
\hline \multirow{8}{*}{$\begin{array}{l}\text { Alta } \\
\text { tecnología }\end{array}$} & 24 & Fabricación de sustancias y productos químicos & 4,1 & 7,0 \\
\hline & 29 & Fabricación de maquinaria y equipo N. C. P. & 5,8 & 5,9 \\
\hline & 30 & Fabricación de maquinaria de oficina, contabilidad e informática & 0,3 & 0,3 \\
\hline & 31 & Fabricación de maquinaria y aparatos eléctricos N. C. P. & 1,9 & 1,9 \\
\hline & 32 & Fabricación de equipo y aparatos de radio, televisión y comunicaciones & 0,2 & 0,4 \\
\hline & 33 & Fabricación de instrumentos médicos, ópticos y de precisión y fabricación de relojes & 1,0 & 0,7 \\
\hline & 34 & Fabricación de vehículos automotores, remolques y semirremolques & 2,8 & 6,7 \\
\hline & 35 & Fabricación de otros tipos de equipo de transporte & 0,8 & 1,0 \\
\hline \multicolumn{3}{|c|}{ Total alta tecnología } & 16,9 & 23,9 \\
\hline
\end{tabular}

Fuente: Elaboración propia, sobre la base de Naciones Unidas, "Clasificación Industrial Internacional Uniforme de Todas las Actividades Económicas, tercera revisión", Informes Estadísticos, serie M, No 4, Rev. 3 (ST/ESASSTAT/SER.M/4/ Rev.3), Nueva York, 1990; datos del Observatorio de Empleo y Dinámica Empresarial; y J. Katz y G. Bernat, "Exit-entry, productivity growth and structural change in response to changes in macroeconomic policy: evidence from Argentina", Innovation and Development, vol. 1, №2, Taylor \& Francis, 2011.

Nota: $\quad$ Datos $=$ entradas + continuadoras - salidas. 